\title{
Scene Construction in Amnesia: An fMRI Study
}

\author{
Sinéad L. Mullally, ${ }^{1}$ Demis Hassabis, ${ }^{2}$ and Eleanor A. Maguire ${ }^{1}$ \\ ${ }^{1}$ Wellcome Trust Centre for Neuroimaging, Institute of Neurology, University College London, London, WC1N 3BG, United Kingdom, and ${ }^{2}$ Gatsby \\ Computational Neuroscience Unit, University College London, London, WC1N 3AR, United Kingdom
}

In recent years, there has been substantial interest in how the human hippocampus not only supports recollection of past experiences, but also the construction of fictitious and future events, and the leverage this might offer for understanding the operating mechanisms of the hippocampus. Evidence that patients with bilateral hippocampal damage and amnesia cannot construct novel or future scenes/events has been influential in driving this line of research forward. There are, however, some patients with hippocampal damage and amnesia who retain the ability to construct novel scenes. This dissociation may indicate that the hippocampus is not required for scene construction, or alternatively, there could be residual function in remnant hippocampal tissue sufficient to support the basic construction of scenes. Resolving this controversy is central to current theoretical debates about the hippocampus. To investigate, we used fMRI and a scene construction task to test patient $\mathrm{P} 01$, who has dense amnesia, $\sim 50 \%$ bilateral hippocampal volume loss, and intact scene construction. We found that scene construction in P01 was associated with increased activity in a set of brain areas, including medial temporal, retrosplenial, and posterior parietal cortices, that overlapped considerably with the regions engaged in control participants performing the same task. Most notably, the remnant of P01's right hippocampus exhibited increased activity during scene construction. This suggests that the intact scene construction observed in some hippocampal-damaged amnesic patients may be supported by residual function in their lesioned hippocampus, in accordance with theoretical frameworks that ascribe a vital role to the hippocampus in scene construction.

\section{Introduction}

The hippocampus plays a pivotal role in supporting autobiographical memory (Scoville and Milner, 1957). In recent years, the importance of the hippocampus has been amplified with the suggestion it also enables the imagining of fictitious and future experiences (Hassabis and Maguire, 2007, 2009; Schacter and Addis, 2007). This idea stems from observations that hippocampaldamaged amnesic patients are unable to construct spatially coherent fictitious or future scenes/events (Tulving, 1985; Tulving et al., 1988; Klein et al., 2002; Hassabis et al., 2007a; Rosenbaum et al., 2009; Andelman et al., 2010; Race et al., 2011; Mullally et al., 2012). This finding is supported by numerous fMRI studies that have documented hippocampal engagement in healthy volunteers when constructing fictitious or future scenes (Okuda et al., 2003; Addis et al., 2007a; Hassabis et al., 2007b; Szpunar et al., 2007).

Collectively, this evidence has generated substantial interest and led to the further observation that a distributed set of brain regions that includes the hippocampus is activated in common during recollection of autobiographical experiences, construction of fictitious/future scenes, and spatial navigation (Buckner

Received Nov. 2, 2011; revised Feb. 13, 2012; accepted March 6, 2012

Author contributions: S.L.M., D.H., and E.A.M. designed research; S.L.M. and E.A.M. performed research; S.L.M. and E.A.M. analyzed data; S.L.M. and E.A.M. wrote the paper.

This work was supported by the Wellcome Trust. We thank P01 and his wife for their time and interest in this research. Thanks also to the Imaging Support team, and Seralynne Vann.

Correspondance should be addressed to Eleanor A. Maguire, Wellcome Trust Centre for Neuroimaging, Institute of Neurology, University College London, 12 Queen Square, London, WC1N 3BG, UK. E-mail: e.maguire@ucl.ac.uk. DOI:10.1523/JNEUROSCI.5522-11.2012

Copyright $\odot 2012$ the authors $\quad 0270-6474 / 12 / 325646-08 \$ 15.00 / 0$ and Carroll, 2007; Hassabis and Maguire, 2007; Schacter and Addis, 2007; Spreng et al., 2009). Moreover, several new theories have been advanced to account for the role of this network in supporting these disparate cognitive functions, all of them emphasizing the crucial role of the hippocampus. For example, the scene construction theory posits that this set of brain areas, and the hippocampus in particular, facilitates the construction of complex spatial contexts or scenes into which event details are bound, and this scene construction process is common to episodic memory, imagination, and navigation (Hassabis and Maguire, 2007; for another account, see Schacter and Addis, 2007). Thus, much is currently invested in using the construction of fictitious/future scenes as leverage to investigate the fundamental operating mechanisms of the hippocampus.

At odds with this view is patient P01 in Hassabis et al.'s (2007a) study [P01 is also known as KN (McKenna and Gerhand, 2002; Aggleton et al., 2005)], who could construct fictitious and personal future scenes despite being densely amnesic (Fig. 1). Other cases have since been reported (Squire et al., 2010, but see Maguire and Hassabis, 2011; see also Maguire et al., 2010a; Cooper et al., 2011; Hurley et al., 2011). This preserved scene construction ability may be evidence of its nonreliance on the hippocampus (Squire et al., 2010). Alternatively, in some patients there may be residual function in remnant hippocampal tissue sufficient to support basic scene construction (Hassabis et al., 2007a; Maguire et al., 2010a; Cooper et al., 2011; Hurley et al., 2011). Resolving this controversy is central to current theoretical debates about the hippocampus. A missing piece of key evidence that could help to adjudicate is the direct examination using fMRI of amnesic patients where scene construction ability is preserved. 
A

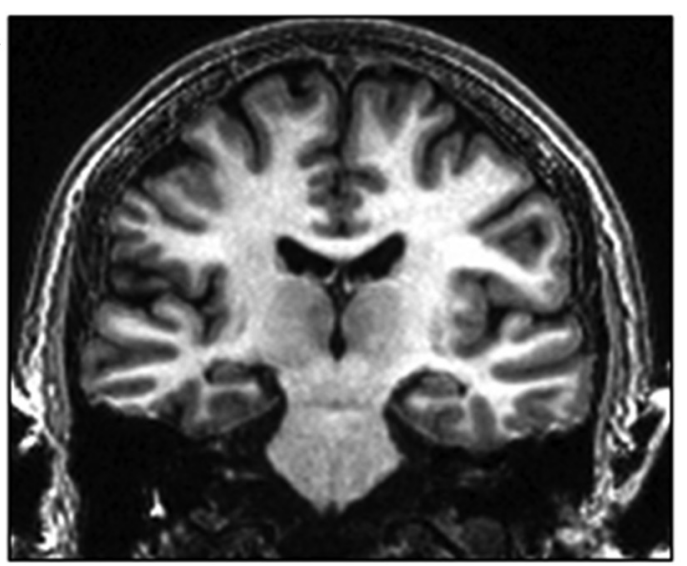

B
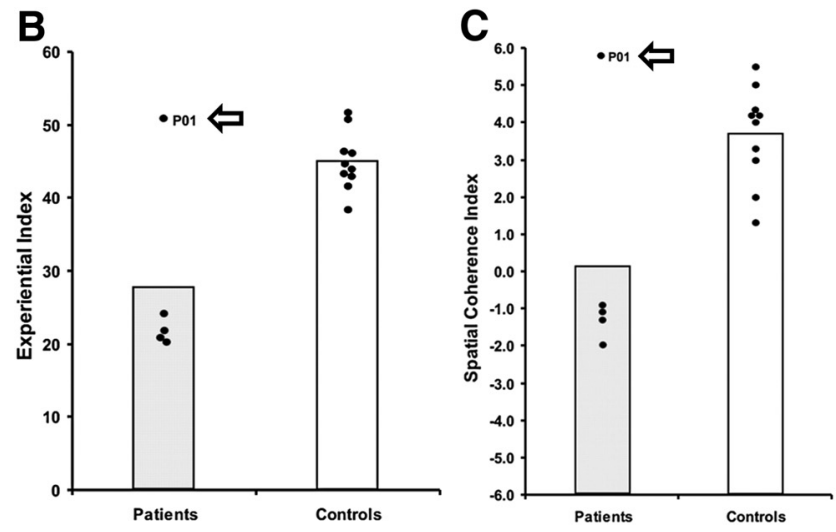

Figure 1. Patient P01. $A$, A coronal view from P01's MRI brain scan. $\boldsymbol{B}$, Scores on the Experiential Index (a measure of the overall richness of the imagined scenes). $C$, Scores on the Spatial Coherence Index (a measure of the spatial contiguousness of the imagined scenes). Data is from the Hassabis et al. (2007a) scene construction task. Each dot represents the data point of a hippocampal-damaged amnesic patient $(n=5)$, and the 10 matched control participants. The data point for P01 is marked with an arrow. Vertical bars signify means for each group. P01 is clearly an outlier, performing similarly to the controls and significantly better than the other hippocampal-damaged amnesic patients.

If theories such as scene construction are correct, then residual hippocampal tissue should be engaged in such patients when they construct novel scenes. To investigate this, we used fMRI and a scene construction task to test patient P01, Hassabis et al.'s (2007a) original anomalous patient, who had dense amnesia and $\sim 50 \%$ bilateral hippocampal volume loss.

\section{Materials and Methods}

Participants. Patient P01, who was 51 years old at the time of testing, has been described in detail previously (McKenna and Gerhand, 2002; Aggleton et al., 2005; Hassabis et al., 2007a). To summarize, this male, righthanded former industrial biochemist contracted meningeo-encephalitis in 1993 at the age of 34 and then recurrent meningitis. He was left without useful motor function below T12, loss of vision in the lower visual field, and severe amnesia. While his structural MRI scans showed bilateral abnormalities in the occipital lobes, the main locus of volume reduction was in the hippocampi [reduced by $48.8 \%$ on the left and $46.2 \%$ on the right; Fig. 1 (Aggleton et al., 2005)]. This reduced hippocampal volume was noted along the anterior-posterior axis of both the left and right hippocampi, coupled with evidence of shortening along this axis, particularly at the head of the hippocampi (Aggleton et al., 2005; their Fig. 2).

On formal neuropsychological tests, P01's full-scale IQ was in the high average range [113, measured by the Weschler Test of Adult Reading (Weschler, 2001)]. He performed normally on tests of language [ $>95$ th percentile, measured by the Category Specific Names Test (McKenna,
Table 1. P01's performance on standard memory tests

\begin{tabular}{lcc}
\hline & Score & Percentile \\
\hline Wechsler Memory Scale III & 77 & 6 \\
$\quad$ Auditory immediate & 61 & 0.5 \\
Visual immediate & 63 & 1 \\
Immediate memory & 52 & 0.1 \\
Auditory delayed & 80 & 9 \\
Auditory recognition delayed & 57 & 0.2 \\
General memory & 131 & 98 \\
Working memory index & & 75 \\
Warrington Recognition Memory Test & $48 / 50$ & 5 \\
Words & $37 / 50$ & \\
Faces & & 25 \\
The Doors and People Test & 17 & $25-75$ \\
Recognition: names & 19 & 1 \\
Recognition: doors & 12 & 1 \\
Recall: people & 22 & \\
Recall: shapes & & \\
\hline
\end{tabular}

1997)], executive function [measured by the Behavioral Assessment of the Dysexecutive Syndrome (BADS): Rule Shift Cards subtest 1 error; the Action Programme subtest 0 errors; the Key Search Test subtest 0 errors; and a verbal fluency task where he generated 17 words beginning with 's' in $1 \mathrm{~min}$ ], and working memory (98th percentile; Table 1). Visual perception was tested using the Visual Object and Space Perception Battery (Warrington and James, 1991). Despite his lower visual field deficit, his visuospatial appreciation was entirely unimpaired (Number Location subtest $=20 / 20)$. His shape perception appeared mildly impaired, but otherwise he performed in the mid-to-low range of normal: Incomplete Letter subtest 14th percentile; Progressive Silhouettes subtest 54th percentile; Number Location subtest $23^{\text {rd }}$ percentile.

While P01 retained the ability to acquire some new semantic information (McKenna and Gerhand, 2002), his anterograde memory for episodic information was grossly impaired. This deficit was evident from the initial assessments performed 4 months postillness, during extensive reassessment in 1998 (McKenna and Gerhand, 2002), and again in 2003 (Aggleton et al., 2005). At each of these time points, P01's scores consistently revealed severe impairment across a range of memory tasks, and specifically on the recall components. Table 1 summarizes his memory scores from 2003 on the Wechsler Memory Scale III (Wechsler, 1997), Warrington Recognition Memory Test (Warrington, 1984), and the Doors and People Test (Baddeley et al., 1994). The apparent sparing of P01's recognition memory compared with his very impaired recall is discussed in detail in Aggleton et al. (2005). Finally, P01's retrograde memory for autobiographical events was grossly impaired across four decades, with virtually no reliable recollections (as verified by his spouse), while his retrograde memory for personal and general semantics was intact. Notably, P01's neuropsychological status has remained very stable over the years and at the time of the current study his spouse reported no change to either his anterograde or retrograde amnesia.

Our aim was to examine $\mathrm{P} 01$ as a rare single case to ascertain whether his preserved ability to construct fictitious scenes was accompanied by engagement of his remnant hippocampal tissue during fMRI. While this was our central motivation, we also took the opportunity to perform some additional exploratory comparisons between P01 and a group of control participants to examine the wider set of brain areas engaged during scene construction. These controls had a similar background (i.e., university educated), performed the same tasks, in the same MRI scanner, using the same image acquisition parameters, and identical data analysis protocol to that used with P01. Results from these control participants were reported previously by Hassabis et al. (2007b). The controls were younger than P01 $[n=21 ; 10$ males; mean age 24.8 years (SD 3.8 ); age range, $18-31$ years], so any comparisons should be treated with caution. We hypothesized there would be no differences between the controls and $\mathrm{P} 01$ in terms of the wider set of brain areas activated given that he, like the controls, was unimpaired at scene construction. 


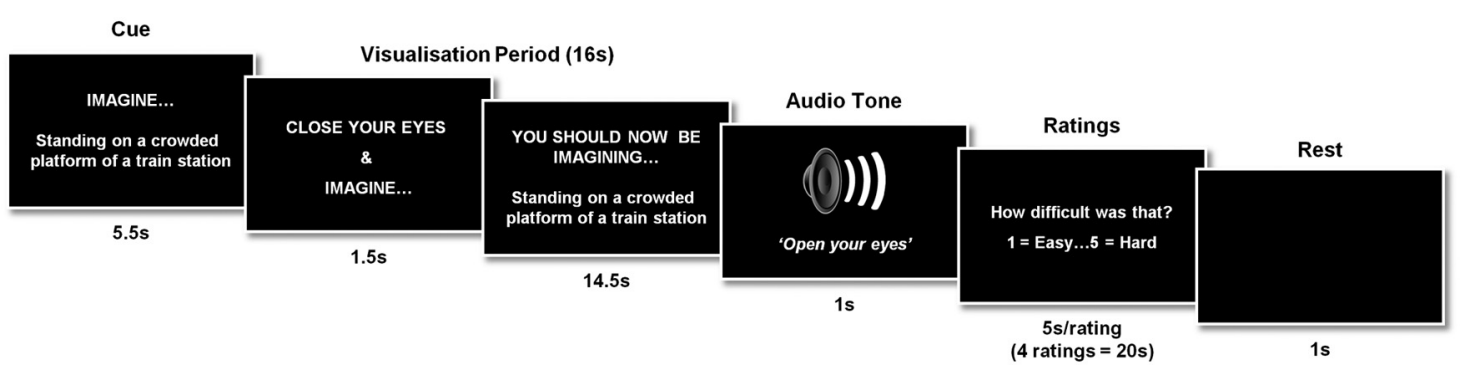

Figure 2. Timeline of an example scene construction trial from P01's fMRI study. See Materials and Methods for full details.

Tasks and procedure. We used the tasks of Hassabis et al. (2007b) with three minor adaptations to assist the patient: the scenario cue appeared on the screen throughout the visualization period, accompanied by the words "You should now be imagining [cue]," in case the patient opened his eyes and could not recall the task (Fig. 2). Similarly, for the ratings, instead of one-word cues being used (e.g. "Difficulty?"), a full question was used ("How difficult was that?"), with an extra $0.5 \mathrm{~s}$ added per rating to allow for additional reading. Just two of the experimental tasks were included from the original Hassabis et al. (2007b) protocol, namely, imagining scenes and single acontextual objects for the first time in the scanner. The other tasks (recall of autobiographical memories/objects and recall of previously imagined scenes/objects) could not be included given P01's amnesia.

The two main experimental conditions (the object condition and the scene condition) had 20 trials each. A baseline control condition was also included ( 10 trials). This yielded a total of 50 trials, which were presented across three scanning sessions. Before scanning, P01 received extensive training to ensure he was thoroughly familiarized with all aspects of the task, including task cues, instructions, timings, and key presses, and to confirm that he could retain the instructions during the scanning session. Training included how to imagine the single, novel objects in the mind's eye (in response to an on-screen cue, e.g., "imagine a spool of bright green thread"), in isolation and against a blank background. The novel, fictitious scenes (e.g., "imagine standing on a crowded platform of a train station") were to be as vivid and life-like as possible, and he was instructed to imagine all of the aspects of the scenes (such as the surrounding environment, how it looked, felt, smelled, and sounded). Throughout the training session, emphasis was placed on constructing novel objects and scenes, and not simply evoking memories of familiar objects or scenes. In the baseline condition, P01 had to imagine a white cross on a black background. Again, detailed instructions and multiple practice trials were given to ensure that he was able to confidently adhere to task requirements.

The trial structure was as follows. The trial cue remained on screen for $5.5 \mathrm{~s}$ and was then replaced by a "close your eyes and imagine" instruction (Fig. 2). At this point, P01 was instructed to close his eyes immediately and begin visualizing the scene or object as vividly as possible. During the $16 \mathrm{~s}$ visualization period, he was required to focus on the scene or object he was imagining, adding more details if necessary. A $1 \mathrm{~s}$ audio tone signaled the end of the visualization period (at which point he had to open his eyes) and the start of the ratings phase. Using an MR-compatible five-button keypad, P01 scored his just-visualized scene or object across four ratings: difficulty [how difficult was that: 1 (very easy) -5 (very hard)], vividness [salience of the imagery: 1 (not vivid)-5 (very vivid)], spatial coherence [contiguousness of the spatial context: 1 (an isolated object) -5 (a contiguous scene)], and memory [how much like a memory the visualized scene or object was: 1 (nothing at all like a memory)-5 (exactly like a memory) ]. He had $5 \mathrm{~s}$ to respond for each rating. This was followed by a $1 \mathrm{~s}$ period of rest before the cue for the next trial was presented. The spatial coherence rating provided an important internal check, enabling us to verify that $\mathrm{P} 01$ had retained the task cue throughout the trial (i.e., if the trial was an object trial, then it should be rated as an isolated object, whereas if the cue described a scene, then the trial should be rated higher and be considered a contiguous scene). The baseline control condition was followed by one rating [i.e., "how focused on the cross did you manage to stay": 1 (not at all focused) -5 (very focused)]. Key task instructions were reinforced between each of the scanning sessions.

Immediately following scanning, P01 was asked what he had been doing during scanning, and was able to report the task instructions correctly. Three of the scene cues and three of the object cues were then presented to $\mathrm{P} 01$, one at a time, and he was asked to imagine them this time out loud to test that his scene construction ability was intact. He was then asked to describe how he went about constructing a scene in his mind's eye. He was also probed about how difficult and effortful he found scene construction.

Behavioral data analysis. Data are presented as mean values \pm SD. Statistical significance was calculated by looking at differences in the ranked position order of P01's ratings for the scene trials and the object trials (Mann-Whitney $U$ test). All tests performed were two-tailed and differences were considered statistically significant at $p<0.05$.

Scanning parameters and preprocessing. T2*-weighted echo planar images (EPI) with blood oxygen level-dependent (BOLD) contrast were acquired on a 1.5 tesla Siemens AG Sonata MRI scanner. Scanning parameters were selected to achieve whole-brain coverage: 45 oblique axial slices angled at $30^{\circ}$ in the anterior-posterior axis, $2 \mathrm{~mm}$ thickness $(1 \mathrm{~mm}$ gap), repetition time $4.05 \mathrm{~s}$, slice time $90 \mathrm{~ms}$, TE $50 \mathrm{~ms}$, field of view 192 $\mathrm{mm}, 64 \times 64$ matrix, in-plane resolution $3 \times 3 \mathrm{~mm}$. The first six dummy volumes from each session were discarded to allow for T1 equilibration effects. Field maps were acquired with a standard manufacturer's double echo gradient echo field map sequence (short TE, $10 \mathrm{~ms}$; long TE, 14.76 $\mathrm{ms}$; whole-brain coverage; voxel size, $3 \times 3 \times 3 \mathrm{~mm}$ ). A T1-weighted structural scan was also acquired with $1 \mathrm{~mm}$ isotropic resolution. Data were analyzed using the statistical parametric mapping software SPM8 (www.fil.ion.ucl.ac.uk/spm). The Hassabis et al. (2007b) control participants' data were reanalyzed in SPM8 to allow for direct comparison with the data of P01. Spatial preprocessing consisted of realignment and unwarping (using field maps), normalization to a standard EPI template in Montreal Neurological Institute (MNI) space with a resampled voxel size of $3 \times 3 \times 3 \mathrm{~mm}$, and smoothing using a Gaussian kernel with full width at half maximum of $8 \mathrm{~mm}$.

fMRI data analysis. After preprocessing, statistical analysis was performed using the general linear model. The experiment had two main imagining conditions (i.e., objects and scenes) and one baseline control (fixation cross) condition. We modeled the time period from the start of the visualization period (i.e., from the "close your eyes and imagine" cue) until the end of the visualization period as a boxcar function of $16 \mathrm{~s}$ duration. This was convolved with the canonical hemodynamic response function to create regressors of interest. P01's movement parameters were included as regressors of no interest and the subject-specific parameter estimates pertaining to each regressor (betas) were calculated for each voxel. First level contrasts were performed on these parameter estimates. As in Hassabis et al. (2007b), we report the fMRI results at a voxel-level threshold of $p<0.001$ whole-brain uncorrected (minimum cluster size of five voxels). We report all areas activated at this threshold.

A formal comparison of P01's fMRI data with that of the control participants was also performed. As this analysis sought to compare activity in a single patient to that observed in a group of participants $(n=$ 21 ), we adapted a statistical approach designed for the comparison of a single case in neuropsychology to a reasonably small sample of control 


\section{Cue: Imagine that you are lying on a deserted white sandy beach in a beautiful tropical bay}

P01: The sand is hot under the touch. It's beautifully white. It's so white that it looks like it has been painted white. And the sea is brilliant blue. The waves... you can hear the waves crashing against the beach and stirring it up down below, and there are big waves, waves that you could surf on if you had a surf board. Around, or to the back of me, there are palm trees, and in the palm trees there are coconuts hanging. There are monkeys climbing up the trees, trying to get the coconuts from the trees. On the beach are fabulous stones and pebbles, ones that I have never seen in my life before. They are brilliant colours. And they have been polished by the sea... they've been really polished. And when they get wet, when the sea is crashing up against them, they are shining, all different colours... The sand is so white, it's unbelievable. It's like flour. And it's really fine to walk on. And it feels nice underfoot. And the heat of the sand where it's been baked by the sun... There is not a cloud in the sky. It's really, really sunny... ever so hot. And there's barely any shade anywhere. I'm the only person on the beach. And if you look right the way along, from one side to the other, all you can see is the sea and the beach. Just in a straight line, and nothing else. And if you look behind you, all you can see are plants... tropical plants. Like ones you have in the house, like cheese plants... that sort of thing, big green leafy things, all growing at the back of you. And you can hear animals living in the forests, monkeys screeching, birds of paradise... all making strange, strange noises, not like your average sparrow, but really sort of cooing. And it's so noisy, all this animal activity going on, and the sea crashing away. And yet there's nobody else on the beach at all. And you can just look to one side, and there's no evidence of any other human, and you look to the other side, and again, no evidence of anybody else.

Con: A sandy beach...presumably it's the day, so the sand will be hot. The sun is beating down. You get the visual illusion of the hot air coming off the sand. There aren't very many insects because it's too hot. Under the sand, you can see the sand gradually moving where the insects are burrowing underneath. In the back there's palms trees... a forest... a tropical sort of forest. With some grass, and flowers, and vines at the edges, turning into the dense trees... and coconut trees of course. It's a nice curved beach that comes to uh... in the far distance... a fairly smooth headland. And on the other end, there's a rather craggy headland, a really steep cliff. One of them is actually quite sort of low and rounded, and then you've got the craggy one with the rocks just off the shore where the waves are breaking. And this is a tropical island, it's in the middle of the ocean, so it's actually quite windy.... so you can hear and feel the wind. When it's really strong, you even feel the sand blowing on you, and possibly also the water from the waves... from the spray. At that point, you can also hear the trees swaying, and see and hear the waves. You also hear the insects in the jungle. Not in the sand but in the jungle. You also hear the sounds of birds, birds calling, and possibly the rustle of animals. If you're unlucky, possibly the roar of a tiger! There's also a hut. One made from wood that's been salvaged from the shore, and thatched with leaves from the trees and grass from the jungle.

Figure 3. An excerpt from P01's performance on the scene construction task. Below is an excerpt from an age-, sex-, and IQ-matched control participant for the same scene.

participants, i.e., a modified $t$ test (Crawford and Howell, 1998; Crawford and Garthwaite, 2002). This treats an individual patient as a sample, and was implemented in SPM using a two-sample $t$ test in which the variance associated with the controls and P01 was assumed to be equal.

\section{Results}

Behavioral data

Ratings

During scanning, after each visualization period, P01 rated the imagined objects and scenes on a five-point scale for four measures (difficulty, vividness, coherence, and similarity to a mem- ory). In terms of trial difficulty, there was no difference between scene and object trials (object trials: mean $=1.4, \mathrm{SD}=1.23$; scene trials: mean $=1, \mathrm{SD}=0.0 ; U=180, Z=-1.43, p=0.15$ ), with both being performed easily. P01 was able to imagine objects and scenes with equal and high vividness (object trials: mean $=5$, $\mathrm{SD}=0$; scene trials: mean $=4.95, \mathrm{SD}=0.22 ; U=190, Z=0.32$, $p=0.32$ ). Importantly, $\mathrm{P} 01$ rated the scene trials as coherent scenes $($ mean $=5, \mathrm{SD}=0.0)$ and the object trials as significantly less scene-like (mean $=1, \mathrm{SD}=0.0 ; U=0, Z=-6.25, p<$ 0.001 ), suggesting that he had retained the task instructions and 
was able to recall the cue beyond the $16 \mathrm{~s}$ visualization period. $\mathrm{P} 01$ rated his constructed scenes and imagined objects as relatively dissimilar from actual memories (object trials: mean $=1.4, \mathrm{SD}=$ 1.23; scene trials: mean $=2.9, \mathrm{SD}=2.01$ ), with the scene trials being rated as more like a memory than the object trials $(U=$ $98, Z=-2.76, p<0.05)$. Finally, P01 reported maintaining a good level of focus throughout the baseline trials (mean = $3.86, \mathrm{SD}=1.46$ ).

\section{Debriefing}

Three of the scene cues and three of the object cues were presented to P01 after scanning and, one at a time, and he was asked to imagine them out loud. His performance, just as it had been when tested by Hassabis et al. (2007a) (Fig. 3), was excellent, confirming the preserved nature of his scene construction. P01 noted that "most of the scene comes in one shot" and described the addition of the extra details as akin to "coloring in a color book." He noted that he does not find scene construction a difficult or effortful process but something that comes quite naturally to him. However, he was unable to remember the constructed scenes even shortly afterward, so that if he was given the same cue again, he constructed an entirely new scene, and was unable to verify whether it had any resemblance to the previously constructed scene (for a similar finding, see Cooper et al., 2011).

\section{Neuroimaging data}

To appreciate the brain areas engaged when P01 constructed novel fictitious scenes, we compared activity associated with the imagination of these scenes relative to the imagination of single acontextual objects (i.e., scenes $>$ objects). Increased activity levels were observed in the right hippocampus, right parahippocampal gyrus, left retrosplenial cortex, and bilateral posterior parietal cortex (Table 2; Fig. 4A). This analysis therefore revealed many components of the scene construction network previously identified in a set of 21 healthy control participants performing the same tasks (Table 2; Fig. 4B) (Hassabis et al., 2007b). Most notably, and of central concern in this study, the remnant of P01's right hippocampus was clearly engaged during scene construction.

As noted above, $\mathrm{P} 01$ rated the scene trials as significantly more like memories than the object trials. However given P01's severe amnesia, we believe it unlikely that his ratings on this question actually mean that the scene trials included a significant memory component. However, to explicitly rule this out, we reanalyzed the fMRI data using only the trials that P01 rated as "nothing at all like a memory." Despite the reduced power associated with this analysis, we continued to observe robust activation within P01's right hippocampus $(33,-28,-14 ; Z=3.66$; $p<0.001$ wholebrain uncorrected), indicating that any potential episodic recall associated with the scene trials (no matter how unlikely) was not driving the activity in P01's right hippocampus.

We performed some additional exploratory analyses comparing P01 to a group of (younger) control participants. To identify which of P01's activation clusters lay within the controls' scene construction network, we created a mask of the data acquired from the controls in the Hassabis et al. (2007b) study (for the same contrast: newly imagined fictitious scenes $>$ newly imagined objects; Fig. 4B). When P01's data were overlaid on the control mask, as anticipated, this illustrated the similar pattern of results between the set of regions supporting P01's scene construction and that of controls (Fig. 5) in right parahippocampal gyrus, left retrosplenial cortex, and bilateral posterior parietal cortex. P01's right hippocampal activity, which is clearly evident (Fig. 4A), did not overlap with control participants' cluster of
Table 2. fMRI results

\begin{tabular}{|c|c|c|}
\hline Region & Peak coordinate $(x, y, z)$ & $Z$ \\
\hline \multicolumn{3}{|l|}{ P01 } \\
\hline \multicolumn{3}{|l|}{ Scenes $>0$ 0bjects } \\
\hline Right superior frontal sulcus & $18,59,19$ & 3.41 \\
\hline Right hippocampus & $36,-28,-14$ & 4.73 \\
\hline Right parahippocampal gyrus & $33,-46,-5$ & 4.52 \\
\hline \multirow[t]{2}{*}{ Left retrosplenial cortex } & $-15,-58,16$ & 3.83 \\
\hline & $-9,-61,10$ & 3.29 \\
\hline \multirow[t]{2}{*}{ Right posterior parietal cortex/angular gyrus } & $45,-70,28$ & 3.76 \\
\hline & $39,-82,31$ & 3.32 \\
\hline Left posterior parietal cortex/angular gyrus & $-33,-85,37$ & 5.76 \\
\hline \multicolumn{3}{|l|}{ Objects $>$ Scenes } \\
\hline Right lateral prefrontal cortex & $48,44,28$ & 3.49 \\
\hline Right intraparietal sulcus & $51,-40,52$ & 4.21 \\
\hline Left intraparietal sulcus & $-54,-40,40$ & 3.87 \\
\hline Right lateral occipital cortex & $48,-52,-17$ & 4.53 \\
\hline Left lateral occipital cortex & $-51,-70,-11$ & 4.71 \\
\hline \multicolumn{3}{|l|}{ Control participants* } \\
\hline \multicolumn{3}{|l|}{ Scenes $>$ 0bjects } \\
\hline Ventromedial prefrontal cortex & $3,24,-9$ & 4.27 \\
\hline Right superior frontal sulcus & $27,27,45$ & 4.42 \\
\hline Right middle temporal cortex & $57,-6,-24$ & 3.70 \\
\hline Right hippocampus & $21,-24,-12$ & 3.86 \\
\hline Left parahippocampal gyrus & $-18,-36,-15$ & 4.28 \\
\hline Right parahippocampal gyrus & $33,-42,-12$ & 4.43 \\
\hline Left retrosplenial cortex & $-12,-60,9$ & 6.08 \\
\hline Right retrosplenial cortex & $12,-57,15$ & 5.52 \\
\hline Right precuneus & $9,-57,48$ & 3.91 \\
\hline Left posterior parietal cortex & $-48,-78,24$ & 4.75 \\
\hline Right posterior parietal cortex & $45,-66,24$ & 4.75 \\
\hline Medial posterior parietal cortex & $9,-75,57$ & 4.73 \\
\hline \multicolumn{3}{|l|}{ Objects $>$ Scenes } \\
\hline Right lateral prefrontal cortex & $42,50,19$ & 3.78 \\
\hline Right intraparietal sulcus & $51,-31,43$ & 4.74 \\
\hline Left intraparietal sulcus & $-54,-31,43$ & 5.30 \\
\hline Right lateral occipital cortex & $42,-64,-8$ & 4.01 \\
\hline Left lateral occipital cortex & $-45,-67,-8$ & 4.29 \\
\hline
\end{tabular}

$p<0.001$ (uncorrected). *Data from Hassabis et al. (2007b). Note that the 0 bjects $>$ Scenes contrast reported here for the control participants [unlike the contrast reported in Hassabis et al. (2007b)] is restricted to items newly imagined in the scanner, in order to be identical to the tasks performed by $\mathrm{P} 01$.

activity in right hippocampus. However, this observation must be considered in the context of P01's gross hippocampal atrophy (46.2\% volume loss along the length of the right hippocampus) and shortening at the anterior end, which renders it difficult to draw comparison between the locations of activity within P01's damaged right hippocampus and that observed in the hippocampi of healthy controls.

We also directly compared the data of P01 with that of the controls from Hassabis et al. (2007b). In line with the overlap analysis above, $\mathrm{P} 01$ activated a region of the right hippocampus more than the control participants $(26,-28,-11, Z=3.27)$, and not any other regions. The controls did not activate any brain areas more than P01. This shows that P01, like control participants (and despite their younger age), activated the wider network for scene construction, even if some areas did not reach the statistical threshold of $p<0.001$ in the original analysis (Fig. 4; Table 2$)$. Indeed when a more liberal threshold $(p<0.005)$ was applied to P01's data, areas such as ventromedial prefrontal cortex $(3,47,-17 ; Z=2.94)$, evident in controls but not initially in $\mathrm{P} 01$, begin to emerge.

Hassabis et al. (2007b) also reported the brain regions that showed increased activity for imagining single acontextual objects compared with scenes, and these included lateral occipital cortex bilaterally, intraparietal sulcus bilaterally, and right lateral 
A
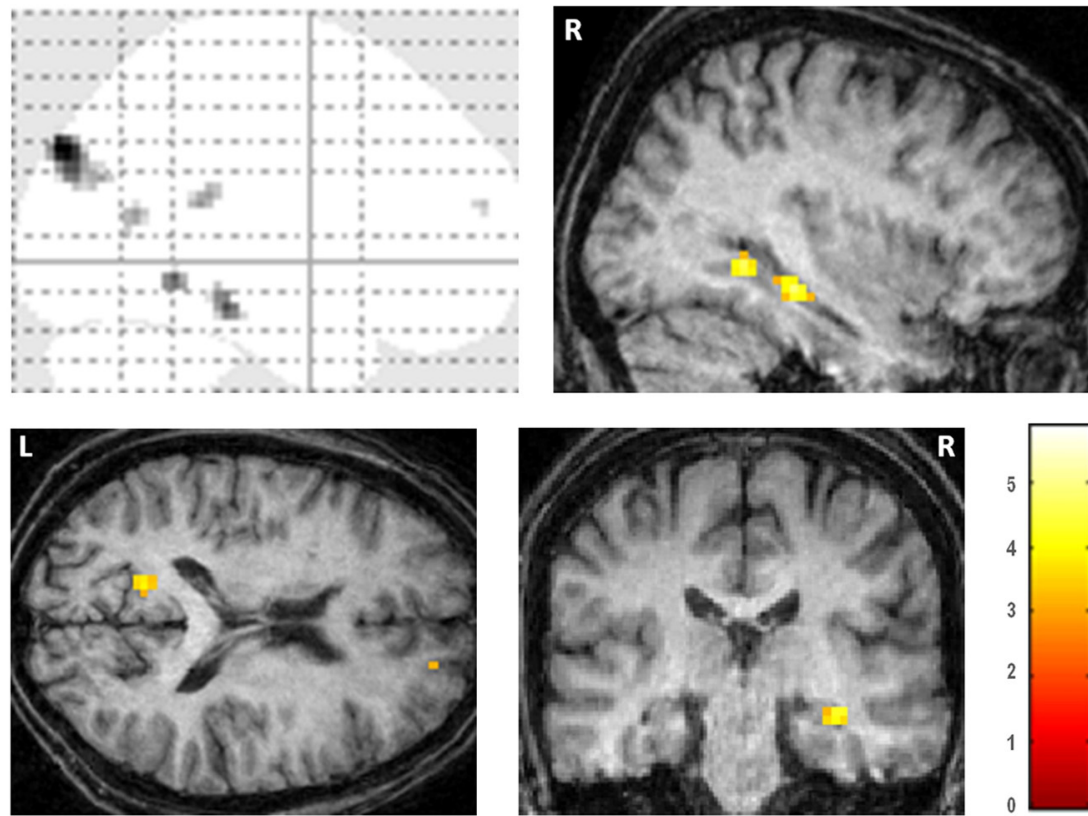

B
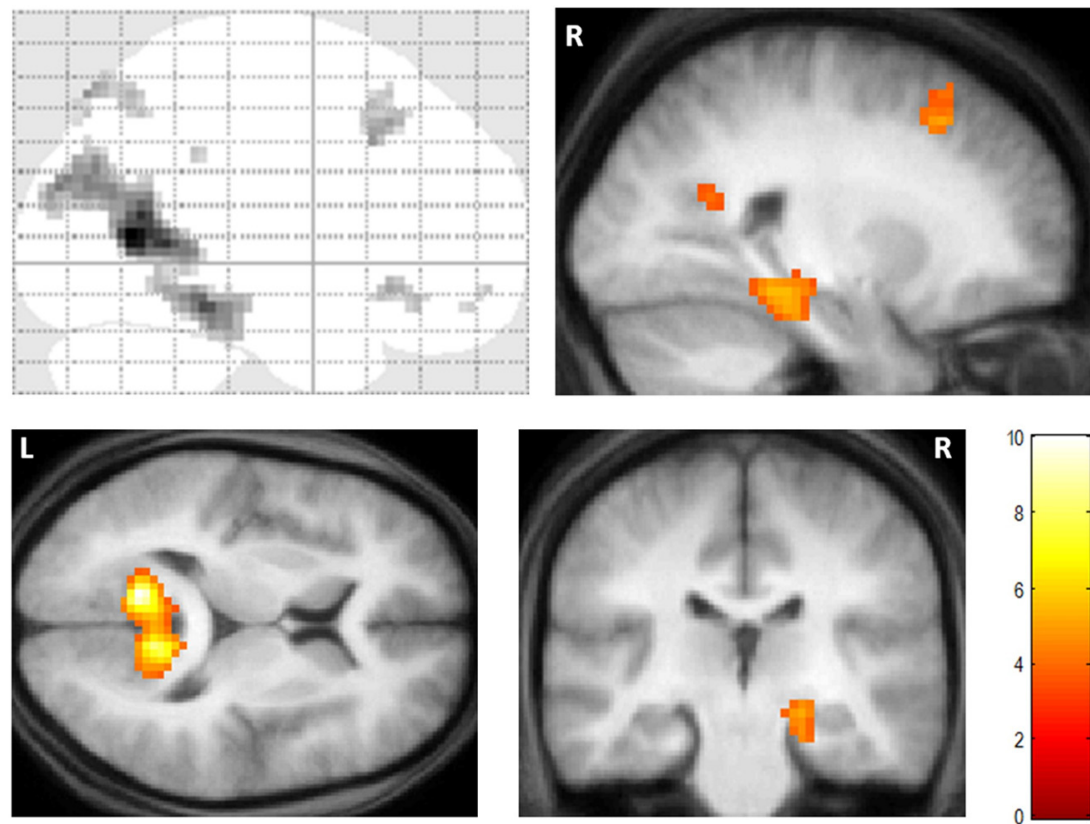

Figure 4. fMRI results. $\boldsymbol{A}$, Brain areas more active for constructing fictitious scenes compared with imagining single acontextual objects in patient P01. Upper left, Sagittal image from a "glass brain," which enables one to appreciate activations at all locations and levels in the brain simultaneously. Activations are shown on sagittal (upper right), axial (lower left), and coronal (lower right) images from P01's structural scan at a threshold of $p<0.001$ (whole brain, uncorrected). The color bar indicates the $z$-scores associated with each voxel. L, Left side of the brain, $R$, right side of the brain. $\boldsymbol{B}$, The same contrast in 21 healthy participants [data from Hassabis et al. (2007b)] shown on the averaged structural MRI scan of those participants.

prefrontal cortex (Table 2). We also examined this contrast in P01 and found that the same areas were engaged (Table 2). This further underscores the similarity of P01's activation patterns to those of the controls, even though they were younger.

\section{Discussion}

Most patients with bilateral hippocampal damage and dense amnesia cannot construct fictitious or future scenes. This makes the rare patients who retain this ability of theoretical importance.
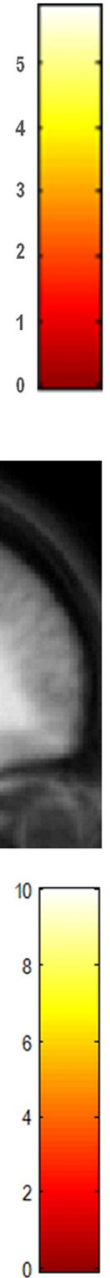

The aim of this study was to examine such a patient, P01 (Hassabis et al., 2007a), who, despite dense amnesia, retained a capacity for scene construction. We used fMRI to investigate the brain areas that were engaged while he successfully constructed fictitious scenes. The main question was whether his grossly atrophied hippocampi (each with the volume reduced by $\sim 50 \%$ ) would be activated. The remnant of $\mathrm{P} 01$ 's right hippocampus exhibited increased activity during scene construction. We also found that scene construction in P01 was associated with increased activity in a set of brain areas that included medial temporal, retrosplenial, and posterior parietal regions, and this overlapped considerably with the network engaged in control participants performing the same task.

The evidence from P01 suggests that the intact scene construction ability observed in some rare hippocampaldamaged amnesic patients may be supported by residual function in their remaining hippocampal tissue (Hassabis et al., 2007a). This accords with theoretical frameworks that ascribe a vital role to the hippocampus in scene construction (Hassabis and Maguire, 2007; Schacter and Addis, 2007), but is at odds with the view that construction of fictitious or future scenes or events occurs independently of the hippocampus (Squire et al., 2010). Of course, activation of a brain region during an fMRI scan does not imply that region is necessary for task performance. However, P01's gross hippocampal atrophy, if anything, would militate against activation. Moreover, he also performed the tasks without difficulty during scanning (and during postscan debriefing), as evidenced by his appropriately high ratings of spatial contiguousness for scene trials compared with low ratings for the object trials. In addition, P01's increased right hippocampal activity persisted when trials rated as similar to a memory were removed from the analysis, suggesting that this region was responding to scene construction rather than episodic recall. Overall, therefore, the most parsimonious conclusion is that P01's activations were not random, and that the engagement of his right hippocampus was related to his preserved scene construction ability. Further evidence for this comes from the laterality of his hippocampal activation. Control participants who imagine fictitious scenes (Hassabis et al., 2007b) or personal future scenarios (Addis et al., 2007a, 2011; Weiler et al., 2010) tend to activate the right more than the left hippocampus. P01's hippocampal activation was also on the right during scene construction, in line with the pattern in control participants (Hassabis et al., 2007b), although we note that the controls here were younger than P01. 

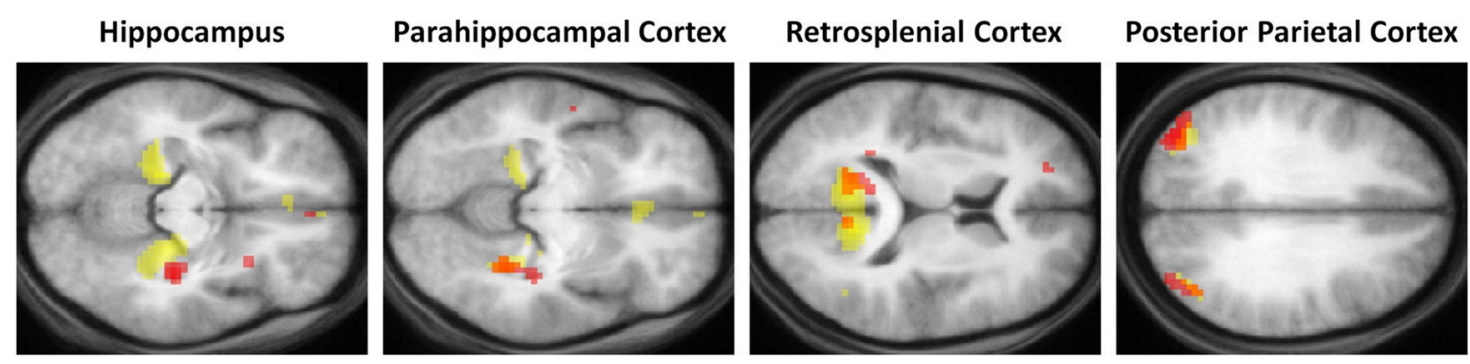

Figure 5. Scene construction activations: overlap between P01 and control participants. Red blobs are activations from P01, yellow blobs are the activations for the control participants from Hassabis et al. (2007b) (who were younger than P01), and orange blobs are where they overlap. Data are shown at $p<0.005$ for display purposes.

It has been suggested that the anterior (right) hippocampus is a key area serving simulations (Addis et al., 2007a, 2011; Schacter and Addis, 2009; Weiler et al., 2010; Martin et al., 2011). The hippocampal activation in $\mathrm{P} 01$ is mid-posterior in terms of peak coordinate and extent. This is in line with the prevailing view that the posterior hippocampus is involved in spatial processing, in this case supporting the spatial backdrop for the constructed scenes (Moser and Moser, 1998; Maguire et al., 2000; Hassabis et al., 2007a). However, any intrahippocampal considerations in this instance must be treated with caution, because while it is possible to assign coordinates in stereotactic space to fMRI activations, what exactly this means for a grossly atrophied hippocampus is not certain. P01's atrophy is along the entire length of the hippocampus, with additional evidence of shortening along the anterior-posterior axis, particularly at the head of the hippocampi (Aggleton et al., 2005). Attempting to infer localization of function within the hippocampus in this context may be futile, as anterior/posterior distinctions might not now be observed. Nevertheless, what is notable is just how much hippocampal volume loss can occur and still the remnant tissue can exhibit task-related activity. In neuropsychology, it is typically assumed that volume loss of the magnitude of P01's renders the hippocampi completely dysfunctional (Gold and Squire, 2005). However, the evidence from P01 and other studies that have also reported fMRI activations in obviously atrophic hippocampal and other tissue during memory tasks (Maguire et al., 2001, 2010b; Rosenbaum et al., 2007; Bowles et al., 2011) challenge this view.

While P01 was able to construct scenes successfully, the other patients reported by Hassabis et al. (2007a) could not. It might be interesting to scan those patients while they attempt to construct scenes, and make a comparison with P01. However, the patients other than P01 had markedly impaired scene construction ability, and across-the-board negative scores for spatial coherence, indicating extreme fragmentation of their internal representation of scenes (Fig. 1) (Hassabis et al., 2007a). Because they are intelligent and articulate people, they were able to list some relevant details associated with the scene cue. Despite being able do this, they had no internal representation of scenes. This finding has since been replicated and extended in a new group of amnesic patients (Mullally et al., 2012). In this latter study, the patients also provided explicit feedback on their attempts to construct scenes, including: "There is no scene in front of me here. It's frustrating because I feel like there should be. I feel like I'm listening to the radio instead of watching it on the TV. I'm imagining different things happening but there's no visual scene opening out in front of me." Thus, based on the objective scene construction measures and the patients' self-declared problem, it is clear that despite knowing what was likely to be there, patients could not construct scenes at all, and consequently had no inter- nal representation of scenes. This is, therefore, not a graded impairment; but a complete inability to construct and visualize scenes. If we scan these patients while they cannot perform scene construction, we simply have no idea what they are doing, and cannot link fMRI activity to anything task-related. As such, the data (whether this involves hippocampal activity or not) would be uninterpretable (Price and Friston, 1999; Price et al., 2006). Thus, while conceptually desirable to scan the impaired patients, this cannot provide meaningful results.

Beyond the hippocampus, P01 activated much of the distributed brain network widely associated with scene construction and future-thinking (Spreng et al., 2009). When compared directly with the Hassabis et al. (2007b) control participants in an exploratory analysis (as they were younger), P01 did not activate any additional areas, suggesting that his preserved scene construction performance was not bolstered by compensatory upregulation of scene construction regions, or the recruitment of any additional brain areas. Similarly, the opposite contrast showed no extra areas activated for control participants compared with P01, highlighting the close correspondence between the brain areas engaged in controls and P01 during successful scene construction.

This study allows us to conclude that some rare patients with bilateral hippocampal damage and amnesia may be able to construct novel fictitious and future scenes via intact mechanisms in the remnant of their right hippocampus. This affirms the close relationship between the hippocampus and scene construction (Hassabis and Maguire, 2007, 2009). It also shows that scene construction, while necessary for autobiographical memory, is not sufficient. P01 has intact scene construction ability but is profoundly amnesic for all new and previous experiences. The scene construction theory posits that while a key function of the hippocampus is scene construction, other processes and other brain areas on top of this are required for effective episodic/ autobiographical memory (Hassabis and Maguire, 2007, 2009). This may involve the additional engagement of the left hippocampus, the upregulation of medial frontal or medial parietal regions (Hassabis et al., 2007b), or particular modes of connectivity across the memory network that get disrupted by bilateral hippocampal damage (Maguire et al., 2001; Addis et al., 2007b).

We propose that if a patient with bilateral hippocampal damage cannot construct scenes, then the knock-on effects of this will include deficits in autobiographical memory, spatial navigation, and simulation of the future, because scene construction is a basic 'must-have' for these functions to operate. Patients like P01, who retain some residual capacity in remaining hippocampal tissue, may be able to construct scenes, but this is not sufficient to rescue their autobiographical memory. What is not possible, we predict, is a situation where a hippocampal-damaged patient has impaired scene construction coupled with fully intact autobio- 
graphical memory (that is stringently measured and involves detailed and vivid reexperiencing of the past), because the latter does not work without the former.

\section{References}

Addis DR, Wong AT, Schacter DL (2007a) Remembering the past and imagining the future: common and distinct neural substrates during event construction and elaboration. Neuropsychologia 45:1363-1377.

Addis DR, Moscovitch M, McAndrews MP (2007b) Consequences of hippocampal damage across the autobiographical memory network in left temporal lobe epilepsy. Brain 130:2327-2342.

Addis DR, Cheng T, Roberts RP, Schacter DL (2011) Hippocampal contributions to the episodic simulation of specific and general future events. Hippocampus 21:1045-1052.

Aggleton JP, Vann SD, Denby C, Dix S, Mayes AR, Roberts N, Yonelinas AP (2005) Sparing of the familiarity component of recognition memory in a patient with hippocampal pathology. Neuropsychologia 43:1810-1823.

Andelman F, Hoofien D, Goldberg I, Aizenstein O, Neufeld MY (2010) Bilateral hippocampal lesion and a selective impairment of the ability for mental time travel. Neurocase 16:426-435.

Baddeley A, Emslie H, Nimmo-Smith I (1994) The doors and people test: a test of visual and verbal recall and recognition. Bury St. Edmunds: Thames Valley Test Company.

Bowles B, O’Neil EB, Mirsattari SM, Poppenk J, Köhler S (2011) Preserved hippocampal novelty responses following anterior temporal-lobe resection that impairs familiarity but spares recollection. Hippocampus 21:847-854.

Buckner RL, Carroll DC (2007) Self-projection and the brain. Trends Cogn Sci 11:49-57.

Cooper JM, Vargha-Khadem F, Gadian DG, Maguire EA (2011) The effect of hippocampal damage in children on recalling the past and imagining new experiences. Neuropsychologia 49:1843-1850.

Crawford JR, Garthwaite PH (2002) Investigation of the single case in neuropsychology: confidence limits on the abnormality of test scores and test score differences. Neuropsychologia 40:1196-1208.

Crawford JR, Howell DC (1998) Comparing an individual's test score against norms derived from small samples. Clin Neuropsychol 12:482-486.

Gold JJ, Squire LR (2005) Quantifying medial temporal lobe damage in memory-impaired patients. Hippocampus 15:79-85.

Hassabis D, Maguire EA (2007) Deconstructing episodic memory with construction. Trends Cogn Sci 11:299-306.

Hassabis D, Maguire EA (2009) The construction system of the brain. Philos Trans R Soc Lond B Biol Sci 364:1263-1271.

Hassabis D, Kumaran D, Vann SD, Maguire EA (2007a) Patients with hippocampal amnesia cannot imagine new experiences. Proc Natl Acad Sci U S A 104:1726-1731.

Hassabis D, Kumaran D, Maguire EA (2007b) Using imagination to understand the neural basis of episodic memory. J Neurosci 27:14365-14374.

Hurley NC, Maguire EA, Vargha-Khadem F (2011) Patient HC with developmental amnesia can construct future scenarios. Neuropsychologia 49:3620-3628.

Klein SB, Loftus J, Kihlstrom JF (2002) Memory and temporal experience: the effects of episodic memory loss on an amnesic patient's ability to remember the past and imagine the future. Social Cognition 20:353-379.

Maguire EA, Hassabis D (2011) Role of the hippocampus in imagination and future thinking. Proc Natl Acad Sci U S A 108:E39.

Maguire EA, Gadian DG, Johnsrude IS, Good CD, Ashburner J, Frackowiak RS, Frith CD (2000) Navigation-related structural change in the hippocampi of taxi drivers. Proc Natl Acad Sci U S A 97:4398-4403.

Maguire EA, Vargha-Khadem F, Mishkin M (2001) The effects of bilateral hippocampal damage on fMRI regional activations and interactions during memory retrieval. Brain 124:1156-1170.

Maguire EA, Vargha-Khadem F, Hassabis D (2010a) Imagining fictitious and future experiences: evidence from developmental amnesia. Neuropsychologia 48:3187-3192.
Maguire EA, Kumaran D, Hassabis D, Kopelman MD (2010b) Autobiographical memory in semantic dementia: a longitudinal fMRI study. Neuropsychologia 48:123-136.

Martin VC, Schacter DL, Corballis MC, Addis DR (2011) A role for the hippocampus in encoding simulations of future events. Proc Natl Acad Sci U S A 108:13858-13863.

McKenna P (1997) The category specific names test. East Sussex: Psychology.

McKenna P, Gerhand S (2002) Preserved semantic learning in an amnesic patient. Cortex 38:37-58.

Moser MB, Moser EI (1998) Functional differentiation in the hippocampus. Hippocampus 8:608-619.

Mullally SL, Intraub H, Maguire EA (2012) Attenuated boundary extension produces a paradoxical memory advantage in amnesic patients. Curr Biol 22:261-268.

Okuda J, Fujii T, Ohtake H, Tsukiura T, Tanji K, Suzuki K, Kawashima R, Fukuda H, Itoh M, Yamadori A (2003) Thinking of the future and past: the roles of the frontal pole and the medial temporal lobes. Neuroimage 19:1369-1380.

Price CJ, Friston KJ (1999) Scanning patients with tasks they can perform. Hum Brain Mapp 8:102-108

Price CJ, Crinion J, Friston KJ (2006) Design and analysis of fMRI studies with neurologically impaired patients. J Magn Reson Imaging $23: 816-826$

Race E, Keane MM, Verfaellie M (2011) Medial temporal lobe damage causes deficits in episodic memory and episodic future thinking not attributable to deficits in narrative construction. J Neurosci 31: 10262-10269.

Rosenbaum RS, Winocur G, Grady CL, Ziegler M, Moscovitch M (2007) Memory for familiar environments learned in the remote past: fMRI studies of healthy people and an amnesic person with extensive bilateral hippocampal lesions. Hippocampus 17:1241-1251.

Rosenbaum RS, Gilboa A, Levine B, Winocur G, Moscovitch M (2009) Amnesia as an impairment of detail generation and binding: evidence from personal, fictional, and semantic narratives in K.C. Neuropsychologia 47:2181-2187.

Schacter DL, Addis DR (2007) The cognitive neuroscience of constructive memory: remembering the past and imagining the future. Philos Trans $\mathrm{R}$ Soc Lond B Biol Sci 362:773-786.

Schacter DL, Addis DR (2009) On the nature of medial temporal lobe contributions to the constructive simulation of future events. Philos Trans R Soc Lond B Biol Sci 364:1245-1253.

Scoville WB, Milner B (1957) Loss of recent memory after bilateral hippocampal lesions. J Neurol Neurosurg Psychiatry 20:11-21.

Spreng RN, Mar RA, Kim AS (2009) The common neural basis of autobiographical memory, prospection, navigation, theory of mind, and the default mode: a quantitative meta-analysis. J Cogn Neurosci 21:489-510.

Squire LR, van der Horst AS, McDuff SG, Frascino JC, Hopkins RO, Mauldin KN (2010) Role of the hippocampus in remembering the past and imagining the future. Proc Natl Acad Sci U S A 107:19044-19048.

Szpunar KK, Watson JM, McDermott KB (2007) Neural substrates of envisioning the future. Proc Natl Acad Sci U S A 104:642-647.

Tulving E (1985) Memory and consciousness. Can Psychol 26:1-12.

Tulving E, Schacter DL, McLachlan DR, Moscovitch M (1988) Priming of semantic autobiographical knowledge: A case study of retrograde amnesia. Brain Cogn 8:3-20.

Warrington EK (1984) Recognition memory test. Windsor: NFERNelson.

Warrington EK, James M (1991) The visual object and shape perception battery. Bury St. Edmunds: Thames Valley Test Company.

Wechsler DA (1997) Wechsler memory scale III. New York: Harcourt.

Wechsler DA (2001) Wechsler test of adult reading. New York: Harcourt.

Weiler JA, Suchan B, Daum I (2010) Foreseeing the future: occurrence probability of imagined future events modulates hippocampal activation. Hippocampus 20:685-690. 